\title{
NOTA CIENTÍFICA \\ EFECTO DE LA TEMPERATURA Y LA LUZ EN LA GERMINACIÓN DE SEMILLAS DE Paspalum guenoarum ${ }^{1}$
}

\author{
MÓNICABEATRIZOTEGUI², MARÍAALEJANDRAPÉREZ ${ }^{3}$, MANOELDE SOUZAMAIA $^{4}$
}

RESUMEN - Paspalum guenoarum es una forrajera recientemente adaptada al subtrópico argentino, con capacidad para cubrir la deficiencia estacionaria de forraje de la región. Para la producción y comercialización de sus semillas, es necesario contar con metodologías adecuadas para el control de calidad de las mismas. El ensayo de germinación constituye una herramienta eficaz para establecer calidad de lotes de semillas, sin embargo hasta el momento no se cuenta con especificaciones precisas para el desarrollo de esta metodología en $P$. guenoarum. La temperatura como la luz son determinantes en la expresión del máximo potencial germinativo, pero las magnitudes y regímenes más favorables deben ser establecidos para cada especie. Por estas razones el objetivo del presente trabajo fue determinar las condiciones de temperatura e iluminación más adecuadas para estimar la calidad mediante el ensayo de germinación en laboratorio. Se utilizó un diseño completamente al azar con arreglo factorial de 2 × 2 con cuatro repeticiones de 100 semillas cada uno. Los factores fueron temperatura constante de $27^{\circ} \mathrm{C}$ y alterna de $20-35^{\circ} \mathrm{C}$ y luz continua y en ciclos de $8 \mathrm{~h} \mathrm{luz} \mathrm{y}$ $16 \mathrm{~h}$ oscuridad. Se determinó el índice de velocidad de germinación (IVG) y el porcentaje de germinación $(\mathrm{PG})$. Los resultados señalan que el régimen de temperatura alterna de $20-35^{\circ} \mathrm{C}$ es el más apropiado para expresar el potencial germinativo, independientemente del régimen lumínico.

Términos para indexación: vigor, semillas forrajeras, analisis de semillas.

\section{EFFECT OF TEMPERATURE AND LIGHT ON Paspalum guenoarum SEEDS GERMINATION}

ABSTRACT - Paspalum guenoarum is a forage species recently adapted to the Argentine subtropical area, which would offer advantages to producers by covering the seasonal pasture deficiency of the region. Adequate methodologies of quality control should be taken into account for seed production and trading. The germination assay is an effective tool to establish the quality of seed lots, but to date, there are no precise specifications for the development of this methodology in $P$. guenoarum. Both temperature and light are determinant in the expression of the maximum germinative potential, but the most favourable magnitudes and regimens must be found for each species. For these reasons, the objective of this study was to establish the adequate temperature and light conditions to evaluate the quality by means of the germination assay in the laboratory. A completely randomised $2 \times 2$ factor design with four replications of 100 seeds was used. Factors were constant temperature at $27^{\circ} \mathrm{C}$ and alternating temperature at $20-35^{\circ} \mathrm{C}$ and continuous light and in cycles of $8 \mathrm{~h}$ light and $16 \mathrm{~h}$ darkness. The germination rate and the germination percentage were determined. The results indicated that the alternating temperature at $20-35^{\circ} \mathrm{C}$ was the most appropriate to express the germination potential, regardless of the light regime.

Index terms: vigor, forage seeds, seeds analysis

\footnotetext{
${ }^{1}$ Submetido em 07/07/204. Aceito para publicação em 03/11/2004. Parte de Tesis de Maestria del primero presentada a Universidad Nacional de Córdoba/Escuela para Graduados, Argentina.

${ }^{2}$ Lic. en Genética, Pos graduada do Facultad de Ciencias Exactas, Químicas y Naturales/Universidad Nacional de Misiones, Argentina, e-mail: motegui@fceqyn.unam.edu.ar
}

\footnotetext{
${ }^{3}$ Ing $^{\mathrm{a}}$ Agr ${ }^{\mathrm{a}}$. MSc. Escuela para Graduados/Facultad de Ciencias Agropecuarias/ UNCdba., Argentina, e-mail: maperez@agro.uncor.edu

${ }^{4}$ Ing $^{\circ} \mathrm{Agr}^{\circ}$, Dr. Profesor Departamento de Fitotecnia, Facultad de Agronomía Eliseu Maciel, Universidade Federal de Pelotas, Brasil, email:maiams@ufpel.tche.br
} 


\section{INTRODUCCIÓN}

Paspalum guenoarum, es una gramínea forrajera nativa del nordeste de Argentina, sur de Brasil y Paraguay. Presenta buena producción de forraje en cantidad como en calidad y relativa tolerancia a heladas y sequías, extendiendo su producción hasta el otoño, cuando otras pasturas nativas son escasas (Urbani et al. 1996).

Para lograr una adecuada implantación se requiere contar con semillas de buena calidad, por lo que es necesario establecer su capacidad de germinación.

Las Reglas Internacionales para el Análisis de Semillas (ISTA, 2004) recomiendan las condiciones del ensayo de germinación para varias especies del género Paspalum no existiendo todavia informaciones para $P$. guenoarum.

Escasos estudios se han realizado en relación a las condiciones necesarias para la germinación de pastos nativos de interés forrajero, particularmente en relación a la magnitud y el régimen de temperaturas como así también a los de iluminación. (Trask \& Pyke, 1998). Las gramineas en general, varían considerablemente en cuanto a los requerimientos de germinación ya sea entre las especies, entre poblaciones de una especie y también dentro de poblaciones.

Mayer \& Poljakoff-Mayber (1989) consideran que la temperatura óptima de germinación es aquella en la que se obtiene el más alto porcentaje de plántulas normales y en el menor tiempo. Para el caso de las poaceas forrajeras tropicales, ésta se encuentra próxima a $35^{\circ} \mathrm{C}$ (Yoon et al. 1985). En cuanto al régimen de temperaturas, la mayoría de las especies que poseen dormición alcanzan porcentajes de geminación más altos cuando se las somete a temperaturas alternas como es el caso de Panicum maximum var. trichoglume (Harty \& Butler, 1975), Bothriochloa laguroides y Chaetotropis elongata (Ferrari, 1999), Briza subaristata (Ferrari et al., 2002), Andropogon hallii (Stubbendieck \& McCully, 1972), Brachiaria humidicola (Carneiro et al., 1986), y Bromus catharticus (Larsen et al., 1973), mientras que aquellas que no se presentan dormidas o han superado este estado, responden mejor con temperaturas constantes, como es el caso de Bromus mollis (Young et al., 1973), Andropogon gerardii, Botriochloa caucasica, Sorghastrum nutans, Panicum virgatum y Digitaria sanguinalis (Hsu et al., 1985).

Las semillas en general necesitan niveles adecuados de oxígeno, humedad y temperatura para germinar, pero muchas especies requieren además determinadas condiciones de iluminación para lograrlo (Copeland \& McDonald, 1995). Se ha comprobado que la luz no influye en forma aislada, sino que es la combinación entre ciertas temperaturas y la luz la que favorece la germinación (Toole, 1976).

La mayoría de las especies de Paspalum necesitan luz para germinar, a excepción de $P$. atratum que germina tanto en luz como en oscuridad (Kalmbacher et al., 1999). Las restantes especies estudiadas hasta el momento: P. plicatulum (Flenniken \& Fulbright, 1987) y P. dilatatum (Schrauf et al., 1995) y las incluidas en las Reglas para el Análisis de Semillas (Brasil, 1992): P. conspersum, P. notatum, P. scrobiculatum, P. urvillei, $P$. wettsteinii manifiestan mejor su potencial germinativo en presencia de luz.

En cuanto al régimen de iluminación Fulbright \& Flenniken (1988), demostraron en distintos cultivares de $P$. plicatulum que la respuesta al fotoperíodo es dependiente de la procedencia geográfica del local de produccion de las semillas.

En el caso de $P$. guenoarum, las Reglas para el Análisis de Semillas de Brasil (1992) recomiendan utilizar luz alterna para el ensayo de germinación, aunque aclaran que es una especie que aún permanece en estudio.

Dado que no se cuenta con información relacionada con las condiciones de germinación más adecuadas para $P$. guenoarum, el objetivo de este trabajo fue estudiar los efectos de la temperatura y de la luz, constantes y alternas, sobre la velocidad y el porcentaje final de germinación.

\section{MATERIAL Y MÉTODOS}

Los ensayos se realizaron con un lote semillas de Paspalum guenoarum cosecha 2000, provenientes del campo experimental de la Facultad de Ciencias Agrarias de la Universidad Nacional del Nordeste, Argentina con tres meses de almacenamiento en condiciones de laboratorio a 17-25 \pm 5 ${ }^{\circ} \mathrm{C}$ y $40-90 \pm 5 \%$ de humedad.

Se trabajó con cuatro repeticiones de 100 semillas por tratamiento sembradas en cajas plásticas sobre papel humedecido con agua destilada. Se determinó el indice de velocidad de geminación (IVG) (Maguire, 1962) y el porcentaje de germinación (PG) de plántulas normales para cuya evaluación se utilizaron los criterios establecidos para el género Paspalum (Bekendam \& Grob, 1979). Los recuentos de germinación se realizaron cada tres días hasta completar los 28 días recomendados por ISTA (2004) para otras especies de Paspalum.

Los tratamientos realizados fueron:

1. luz constante y temperatura constante de $27^{\circ} \mathrm{C}$ 
(LC+TC);

2. luz alterna $8 \mathrm{~h} / 16 \mathrm{~h}$ oscuridad y temperatura constante de $27^{\circ} \mathrm{C}(\mathrm{LA}+\mathrm{TC})$;

3. luz alterna y temperaturas alternas de $16 \mathrm{~h}$ oscuridad a $20{ }^{\circ} \mathrm{C}$ y $8 \mathrm{~h}$ luz a $35^{\circ} \mathrm{C}(\mathrm{LA}+\mathrm{TA})$;

4. luz constante y temperaturas alternas de $20-35^{\circ} \mathrm{C}$ por 16 y $8 \mathrm{~h}$ respectivamente (LC+TA).

El diseño empleado fue completamente aleatorizado. Los resultados de PG se transformaron en arco seno $\sqrt{\% / 100}$ previo al análisis de varianza (ANOVA, Statgraphic, 1997). Debido al efecto significativo de los tratamientos, se empleó el test de comparación de medias de Tukey $(\mathrm{P}<0.05)$. El IVG fue analizado por regresión, las variables utilizadas fueron, $\mathrm{Y}=\mathrm{n}^{\mathrm{o}}$ de plántulas normales registradas en el día $\mathrm{X}$, siendo $\mathrm{X}$ $=$ días desde el inicio del ensayo.

\section{RESULTADOS Y DISCUSIÓN}

Los dos tratamientos que generaron los porcentajes de germinación más altos fueron los que contaban con temperaturas alternas (LC+TA: 64,75\% y LA+TA: 58\%), mientras que LA+TC presentó el valor más bajo y fue diferente al resto de los tratamientos (Figura 1).

En general los tratamientos con TA presentaron mayor valor de germinación respecto a los tratamientos con TC. Es de destacar que la combinación de $\mathbf{L C}+\mathbf{T A}$ benefició además la velocidad de germinación.

En la Tabla 1 se presentan los resultados obtenidos con el análisis de regresión de los IVG. La función que mejor describió el comportamiento de la variable en todos los tratamientos es la recíproca- $\mathrm{X}: \mathrm{Y}=\mathrm{a}+\mathrm{b} / \mathrm{X}$.. Las curvas de regresión se compararon a través del cálculo de los límites de confianza para la pendiente y la ordenada al origen de cada

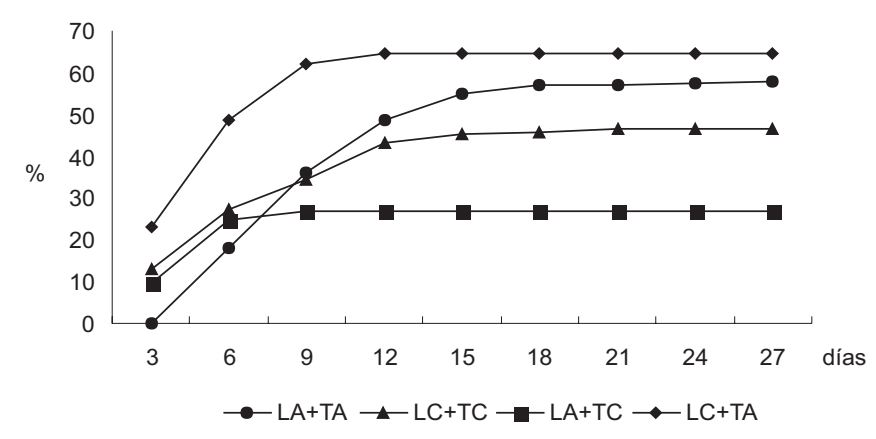

FIGURA 1. Germinación de semillas de Paspalum guenoarum cv. Chané FCA en diferentes condiciones de temperatura e iluminación. una de ellas, encontrándose que solamente LC+TA y LA+TA producen curvas similares. Estos resultados concuerdan con los expresados para PG, indicando que estos dos tratamientos serían los que permiten la mejor expresión de la germinación y que es la temperatura alterna, la condición más importante para que las semillas expresen el máximo potencial germinativo.

La respuesta germinativa observada con respecto al efecto de la luz, está asociado con el fitocromo, que controla la mayoría si no todas las respuestas de las semillas a la luz (Duke, 1985). Así las semillas de $P$. guenoarum que recibieron el tratamiento de luz continua, mantuvieron el balance fotoestacionario más conveniente a la inducción de la germinación que las que recibieron el tratamiento con luz alterna.

La respuesta germinativa observada con respecto a la luz, esta asociado con el fitocromo, que controla la mayoría si no todas las respuestas de las semillas a la luz (Duke, 1985). Así las semillas de P. guenoarum que recibieron el tratamiento de luz continua, mantuvieron el balance fotoestacionario más conveniente a la inducción de la germinación que las que recibieron el tratamiento con luz alterna.

En esta especie en estudio la respuesta fotoperiódica resultó dependiente de la temperatura de acuerdo a lo propuesto por Koller (1971). Al respecto Copeland \& McDonald (1995), sostienen que las especies sensibles a la longitud del día disminuyen este requerimiento si se producen aumentos de temperatura. La alternancia de temperatura en nuestros ensayos fue suficiente para estimular la germinación, independientemente del régimen lumínico. El efecto estimulante de las temperaturas alternas ha sido comprobado en muchas forrajeras tropicales, tales como Agropyron smithii (Toole, 1972), Paspalum plicatulum (Fullbright \& Flenniken, 1988), Paspalum notatum (Marousky \& West, 1988) y Paspalum dilatatum (Schrauf et al., 1995). Al parecer las semillas que responden a la alternancia de este factor presentan mecanismos enzimáticos que funcionan en diferentes temperaturas (Vázquez-Yanes \& Orozco-Segovia, 1987) y esa respuesta se corresponde probablemente, con una adaptación a las fluctuaciones naturales del ambiente (Borges \& Rena, 1993). Por lo que se deduce que la alternancia de temperatura mejora

TABLA 1. Análisis de regresión de los índices de velocidad de germinacion (IVG) alcanzados con cada tratamiento.

\begin{tabular}{ccc}
\hline Tratamiento & R-cuadrado \% & Función \\
\hline LC+TC & 96,22 & $\mathrm{Y}=51,39-121,15 / \mathrm{X}$ \\
LC+TA & 92,43 & $\mathrm{Y}=77,83-166,74 / \mathrm{X}$ \\
LA+TC & 89,32 & $\mathrm{Y}=30,73-56,82 / \mathrm{X}$ \\
LA+TA & 93,31 & $\mathrm{Y}=65,25-212,91 / \mathrm{X}$ \\
\hline
\end{tabular}


el balance y la interacción de las hormonas con las enzimas, los sustratos y el fitocromo preexistente.

Por otra parte en la naturaleza, las fluctuaciones térmicas diarias, típicas de climas tropicales, subtropicales y mediterráneos, provocan un debilitamiento progresivo de las cubiertas duras e impermeables induciendo a la germinación (Bewley \& Black, 1982). Este tipo de respuesta también ha sido demostrado por Thompson et al. (1977), induciendo la germinación de Cenchrus arvensis. Al respecto Taylor (1981) y Fairbrother (1991), sostienen que las fluctuaciones térmicas en condiciones naturales, producen la expansión y contracción de los tejidos de la cubierta seminal provocando fracturas que favorecen la entrada del agua necesaria para la germinación.

La presencia de cubiertas seminales impermeables ha sido estudiada en otras especies de Paspalum (Flenniken \& Fulbright, 1987; Marousky \& West, 1988), de modo que es posible pensar que los mayores porcentajes de germinación alcanzados en $P$. guenoarum con temperaturas alternas se deben al efecto de las fluctuaciones térmicas sobre las cubiertas seminales.

\section{CONCLUSIONES}

La germinación de semillas de Paspalum guenoarum depende de la temperatura y la luz, y es la alternancia de temperatura suficiente estímulo para favorecer tanto el porcentaje de germinación como la velocidad de emergencia.

La alternancia de temperatura $\left(20-35^{\circ} \mathrm{C}\right)$ activa el metabolismo e induce al debilitamiento de las cubiertas impermeables, favoreciendo la imbibición y desencadenando el proceso de germinación.

\section{REFERENCIAS}

BEWLEY, J. D.; BLACK, M. Physiology and biochemistry of seeds in relation to germination. Vol. II. Viability, dormancy and environmental control. Berlin: Springer-Verlag, 1982. 375p.

BORGES, E.E.L.; RENA, A.B. Germinação da sementes. In: AGUIAR I.B.; PIÑA-RODRIGUES, F.C.M.; FIGLIOLIA, M.B. (Ed.) Sementes florestais tropicais. Brasília: ABRATES, 1993. p.83135.

BRASIL. Ministério da Agricultura e da Reforma Agrária. Regras para análise de sementes. Brasília: SNDA/DNDV/CLAV, 1992.365p.

CARNEIRO, J. W. P.; ROSSETO, M. Z.; GIOVANNI, R. Influência da posição da semente no substrato e da temperatura de germinação no desempenho de dois lotes de sementes de Brachiaria humidicola. Revista Brasileira de Sementes, Brasília, v.8, n.3, p.41-46, 1986.
COPELAND, A. D.; MC DONALD, M. B. Seed germination. In: COPELAND, A. D.; MC DONALD, M. B. (Ed.). Principles of Seed Science and Technology. 3 ed. Boston: Kluwer Academic Publishers, 1995. p.59-110.

DUKE, S. O. Reproduction and ecophysiology. In: DUKE, S. O. (Ed.). Weed Physiology. Boca Raton: CRC Press Inc., 1985. p.165.

FAIRBROTHER, T. E. Effect of fluctuating temperatures and humidity on the softening rate of hard seed of subterraneum clover Trifolium subterraneum L. Seed Science and Technology, Zürich, v.19, n.1, p.93-105, 1991.

FERRARI, L. Efectos de la temperatura y de pretratamientos en la germinación de Bothriochloa laguroides (DC.) Herter y Chaetotropis elongata (Knut) Björkman. Revista Brasileira de Sementes, Brasília, v.21, n.2, p.84-87, 1999.

FERRARI, L.; POSTULKA, E. B.; LÓPEZ, C. Condiciones de germinación de semillas de Briza subaristata Lam. recolectadas en diferentes sitios de la pampa deprimida (Argentina). Revista de Investigación Agraria, Serie Producción y Protección Vegetal, INIA, España, v.17, n.2, p.157-162, 2002.

FLENNIKEN, K. S.; FULBRIGHT, T. E. Effects of temperature, light and scarification on germination of brownseed Paspalum seeds. Journal of Range Management, Denver, v. 40, n. 2 p.175$179,1987$.

FULBRIGHT, T. E.; FLENNIKEN, K. S. Causes of dormancy in Paspalum plicatulum (Poaceae) seeds. The Southwestern Naturalist, Kansas, v.33, n.1, p35-39, 1988.

HARTY, R. L.; BUTLER, J. E. Temperature requirements for germination of green panic, Panicum maximum var. trichoglume, during the after-rippening period. Seed Science and Technology, Zürich, v. 3, n.2, p.529-536, 1975.

HSU, F. H.; NELSON, C.; J.; MATCHES, A. G. Temperature effects on germination of perennial warm-season forage grasses. Crop Science, Madison, v.25, n.2, p.215-220, 1985.

INTERNATIONAL SEED TESTING ASSOCIATION. International Rules for Seed Testing. Zürich: ISTA, 2004. 333p.

KALMBACHER, R. S.; WEST, S. H.; MARTIN, F. G. Seed dormancy and aging in atra paspalum. Crop Science, Madison, v.39, n.6, p.1847-1852, 1999.

KOLLER, D. Analysis of the dual action of white light on gemination of Atriplex dimorphostegia (Chenopodiaceae). Israel Journal of Botany, Jerusalem, v.19, sin número, p.499-516, 1971.

LARSEN, A. L.; MONTIGILLION, D. P.; SCHROEDER, E. M. Germination of dormant and nondormant rescuegrass seed on the thermogradient plate. Agronomy Journal, Madison, v.65, n.1, p.56-59. 1973.

MAGUIRE, J. D. Speed of germination-aid in selection and evaluation for seedling emergence and vigor. Crop Science, Madison, v.2, n.2, p.176-177, 1962.

MAROUSKY, F. J.; WEST, S. H. Germination of bahiagrass in response to temperature and scarification. Journal of the American Society for Horticultural Science, Alexandria, v.113, n.6, p.845-849, 1988.

MAYER, A. M.; POLJAKOFF-MAYBER, A. The Germination of Seeds. 2 ed. New York: Pergamon Press Ltd., 1989. 192p. 
SCHRAUF, G. E.; CORNAGLIA, P. S.; DEREGIBUS, V. A.; RÍSSOLA, M. G. Improvement germination behaviour of Paspalum dilatatum Poir. seeds under different pre-conditioning treatments. New Zealand Journal of Agricultural Research, Wellington, v.38, n.3, p.501-509, 1995.

STATGRAPHIC PLUS for Windows 3.0. Serial Number: 3872170. Copyright(C) 1994-1997 by Statistical Graphics Corp. Licensed to: UNaM-LAS.

STUBBENDIECK, J.; MCCULLY, W. G. Factors affecting germination, emergence and establishment of sand bluestem. Journal of Range Management, Denver, v.25, n.5, p.383-385, 1972.

TAYLOR, G. B. Effect of constant temperature treatments followed by fluctuating temperatures on the softening of hard seeds of Trifolium subterraneum L. Australian Journal of Plant Physiology, Melbourne, v.8, n.2, p.547-558, 1981.

THOMPSON, K.; GRIME, J. P.; MASON, G. Seed germination in response to diurnal fluctuations of temperature. Nature, London, v.267, p.147-149, 1977.

TOOLE, V. K. Light and temperature control of germination in Agropyron smithii seeds. Plant Cell Physiology, Oxford, v.17, n.11, p.1263-1267, 1972.

TOOLE, V.K. Effects of light, temperature and their interaction on the germination of seeds. Seed Science and Technology, Zürich, v.1, n.2, p.339-396, 1976.

TRASK, M. M.; PYKE, D. A. Variability in seed dormancy of three Pacific Northwestern grasses. Seed Science and Technology, Zürich, v.26, n.1, p.179-191, 1998

URBANI, M. H.; QUARÍN, C. L.; ESPINOZA, F; NORMAN, G. A. Domesticación de gramíneas apomícticas nativas como forrajeras para el subtrópico húmedo. In: REUNIÓN DE COMUNICACIONES CIENTÍFICAS Y TECNOLÓGICAS, 11., 1996, Corrientes. Resumens... Corrientes: UNNE, 1996. p.81-84.

VÁZQUEZ-YANES, C.; OROZCO-SEGOVIA, A. Fisiología ecológica de semillas en la Estación de Biología Tropical "Los Tuxtlas", Veracruz, México. Revista de Biología Tropical, Costa Rica, v.35, n.1, p.85-96, 1987.

YOON, S.; MURUYAMA, S; KOSAKA, S. Studies on temperature responses of grasses: 2. Comparison on germination of temperate and tropical grasses in various temperature conditions. Journal of the Yamagata Agriculture and Forestry Society, Japan, v.42, n.1, p.21-26, 1985.

YOUNG, J. A.; EVANS, R. A.; KAY, B. L. 1973. Temperature requeriments for seed germination in an annual-type rangeland community. Agronomy Journal, Madison, v. 65, n.3, p. 656-659, 1973. 